\title{
The Southampton Consensus Guidelines for Laparoscopic Liver
}

\section{Surgery: From Indication to Implementation}

Mohammad Abu Hilalc, s, 1, Luca Aldrighetti s, 2, Ibrahim Dagher s, 3, Bjorn Edwin s, 4, Roberto Ivan Troisi s, 5, Ruslan Alikhanove, 6, Somaiah Aroorie, 7, Giulio Bellie, 8, Marc Besselink ${ }^{e, ~ 9, ~ J a v i e r ~ B r i c e n o ~}{ }^{\mathrm{e}}$ 10, Brice Gayete, 11, Mathieu

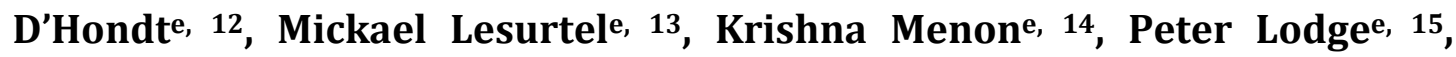
Fernando Rotellare, 16, Julio Santoyo e, 17, Olivier Scattone, 18, Olivier Soubrane 19, Robert Sutcliffee, 20, Ronald Van Dame, 21, Steve Whitee, 22, Mark Hallsi, 1, Federica Ciprianij, 2, Marcel Van der Poeli, 9, Ruben Ciriaj, 10, Leonid Barkhatovi, 4, Yrene Gomez-Luque ${ }^{\mathrm{j}, 10}$, Sira Ocana-Garciaj, 10, Andrew Cook ${ }^{\mathrm{M}}$, 23, Joseph Buellv, 24, Pierre Alain Clavienv, 25, Christos Dervenis ${ }^{v}$, 26, Giuseppe Fusaiv, 27, David Gellerv, 28, Hauke Langv, 29, John Primrosev, 1, Mark Taylorv, 30 , Thomas Van Gulikv, 9, Go Wakabayashiv, 31, Horacio Asbunv, 32*, Daniel Cherquis, 33*

\section{Roles:}

C - Chairman; S - Steering Committee; E - Expert Panel; J - Junior Expert; M Methodologist; V - Validation committee; * - Shared senior authors

Affiliations: 1. University Hospital Southampton, Southampton, United Kingdom; 2. San Raffaele Hospital, Milan, Italy; 3. Antoine-Beclere Hospital, Paris, France; 4. The Intervention Centre, Dept. of HBP surgery, Oslo University Hospital and Institute of Clinical Medicine, Oslo University, Oslo, Norway; 5. Gent University Hospital Medical School, Gent, Belgium; 6. Moscow Clinical Scientific Centre, 
Moscow, Russia; 7. Plymouth Hospital NHS Trust, Plymouth, United Kingdom; 8. Loreto Nuovo Hospital, Naples, Italy; 9. Academic Medical Centre, Amsterdam, Netherlands; 10. University Hospital Reina, Sofia Cordoba, Spain; 11. Institut Mutualiste Montsouris, Paris, France; 12. AZ Groeninge, Kortrijk, Belgium; 13. Croix Rousse University Hospital, Lyon, France; 14. King's College Hospital, London, United Kingdon; 15. Leeds Teaching Hospitals NHS Trust, Leeds, United Kingdom; 16. Navarra University, Pamplona, Spain; 17. Malaga University Hospital, Malaga, Spain; 18. Pierre and Marie Curie University, Paris, France; 19. Beaujon Hospital, Clichy, France; 20. University Hospital Birmingham, Birmingham, United Kingdom; 21. Maastricht University Medical Centre, Maastricht, Nehterlands; 22. Newcastle upon Tyne NHS Foundation Trust, Newcastle, United Kingdom; 23. Southampton Clinical Trials Unit, University Hospital Southampton, Southampton, United Kingdom; 24. Louisiana State University and Medical Center, New Orleans, United States of America; 25. University Hospital Zurich, Zurich, Switzerland; 26. Agia Olga Hospital, Athens, Greece; 27. Royal Free Hospital NHS Trust, London, United Kingdom; 28. University of Pittsburgh, Pittsburgh, United States of America; 29. Mainz University Hospital, Mainz, Germany; 30. Mater hospital, Belfast, Northern Ireland; 31. Iwate Medical University, Morioka, Japan; 32. Mayo Clinic, Jacksonville, United States of America and 33. Hepatobiliary Centre-Paul Brousse Hospital, Villejuif-Paris, France.

\section{With special thanks to:}

\section{Our patient representatives: Mr Paul Over and Mr Tim Guerrier}




\section{Corresponding author}

Prof. Mohammed Abu Hilal, MD PhD DocEur FRCS FACS,

Department of Hepato-Biliary and Pancreatic Surgery

University Hospital Southampton NHS Foundation Trust

Tremona Road, Southampton S016 2YD, UK

Tel: +447863354035

Email: abuhilal9@gmail.com,

\section{Key words:}

Laparoscopic Liver Surgery; Consensus; Guidelines; Clinical Practice;

Southampton; Indication; Patient selection; Procedures; Technique;

Implementation.

\section{Disclosures}

None

\section{Paper information}

Abstract: 245 words

Main article: 4,590 words (excluding tables and graphs)

2 Appendices

1 Table

1 Figure 


\section{Introduction:}

The first European Guidelines Meeting on Laparoscopic Liver Surgery (EGMLLS) was held in Southampton on the February $10^{\text {th }}$ and $11^{\text {th }}, 2017$ with the specific aim of presenting and validating the first set of guidelines for laparoscopic liver surgery (LLS).

Previously, the consensus meeting in Louisville (2008)(1) reviewed the feasibility of LLS while that of Morioka (2014)(2) focused on a comparison with open resections, then the current standard of practice, demonstrating a clear role for the laparoscopic approach in the modern era of liver surgery. While the laparoscopic approach must continue to demonstrate a lack of inferiority compared to the open approach, the future must be directed at its potential advantages, development and safe progression (3). Building on the foundation laid by the two previous meetings this manuscript represents the first set of clinical practice guidelines designed specifically to direct the safe future development of laparoscopic liver surgery. The Southampton Guidelines aim to provide both experienced and training surgeons, and centres, guidance as to the appropriateness of care, to reduce variations in practice and to facilitate the safe expansion of LLS with the goal of improving patient care (4). 


\section{Methodology:}

The members of the steering committee and the expert panel were selected by the committee chairman for their wealth of experience and their significant contributions to the development of laparoscopic liver surgery. Of the eleven members of the international validation committee 7 surgeons only perform open resections while the remaining 4 surgeons perform both open and laparoscopic liver surgery. To provide clear clinical practice guidelines on LLS and its safe expansion, five key domains were identified by the Steering Committee: Indications, Patient selection, Procedures, Techniques and Implementation. Each domain was further subdivided into topics, for example the "Indication" domain was separated into: resections for "Colorectal Liver Metastases", "Hepatocellular Carcinoma" and "Benign and Other Rare Liver Metastases". In addition to the 5 members of the steering committee a further 18 liver surgeons, all with recognised expertise in LLS, were selected to form the Expert Panel. The expert panel was divided into working groups and each was assigned a number of topics to develop specific guidelines. An independent validation committee of 11 experts and 2 patient representatives were involved throughout the process of statement production.

The methodology for the production of the Southampton Guidelines was developed in collaboration with an independent methodologist. A unique approach to the production was adopted by integrating three validated methods: the SIGN (Scottish Intercollegiate Guidelines Network) methodology for the assessment of evidence and development of guideline statements (5); the Delphi method (for establishing expert consensus)(6) and the AGREE II-GRS (Global 
Rating Scale) Instrument (7) for the assessment of the methodological quality and external validation of the final statements.

A systematic review using Ovid Medline and Pubmed was undertaken in July 2016 and repeated in January 2017 to review all the existing literature for each topic. All manuscripts meeting the inclusion criteria were evaluated using the SIGN methodology to establish the Study Quality and assigned an Evidence Level (See Appendix 1 and PRISMA diagram). Through the use of Considered Judgement Forms (as per the SIGN methodology) the findings of the systematic review and the opinions of the experts in each working group were combined to form the provisional statements. A form of recommendation (i.e. strength), based upon the level of evidence from the systematic review, was assigned to each statement (See Appendix 2). All the statements were amalgamated and disseminated to the entire expert panel for voting in accordance with the Delphi methodology. This methodology allows each expert to either agree or disagree with a given statement and make recommendations for changes to that statement should they feel it necessary. If a statement reached greater than or equal to a $95 \%$ agreement in the first Delphi round it was accepted into the guidelines for presentation at the meeting and removed from further Delphi rounds. Statements failing to reach a $95 \%$ agreement were returned to the original working group, along with their respective anonymised comments, for revision and were entered into the subsequent Delphi round. After 3 Delphi rounds 66 statements had reached at least a 95\% agreement and 3 had not. The identities of those producing the provisional statements and those providing feedback remained anonymous except to the guideline's chairman. 
At the pre-meeting assembly, a fourth Delphi round was held with the intent to review the guideline statements and reach a consensus on the three outstanding statements. In addition, the form of recommendations assigned to each statement was reviewed, assessed and modified to ensure the evidence level provided neither over nor under represented the statement. This was performed taking into consideration factors such as the clinical importance of the topic, the relevance of implications to the clinical setting and the consistency of the body of evidence. At this point two statements were removed as they failed to reach agreement; hence 67 statements were accepted for the meeting. The Validation Committee reviewed the literature searches and the subsequent summaries used for the production of the guideline statements, specifically examining the methodological techniques underpinning the production of each statement as per the AGREE II-GRS tool (8).

In addition to the expert panel and validation committee the 2-day conference was attended by over 190 specialists, from 23 different countries, all sharing an interest in liver surgery. During the conference the highest-level evidence supporting each statement was presented along with the form of recommendation. In addition, all in attendance voted electronically demonstrating their additional support, or otherwise, for each statement. The validation committee considered the outcomes of these votes, the opinions of the patient representatives and proposed a number of recommendations before granting endorsement. The expert panel accepted all the recommendations from the validation committee. A detailed description of this novel methodology for the development of surgical guidelines will be published separately, as will the detailed systematic reviews for the core topics. 



\section{Guidelines:}

The Southampton Guidelines were derived from the afore mentioned methodology and thus are based on published evidence and expert opinion. It is of critical importance to note that the majority of the evidence originates from surgeons experienced in both liver surgery and advanced laparoscopic techniques working in specialist liver centres. Therefore the guidelines should not be misconstrued as an endorsement for surgeons to perform LLS without the necessary experience and training or in an institution without the proficiency and support to practice liver surgery. It is also noteworthy that LLS accounts for 30$60 \%$ of liver resections in these specialist centres and therefore there is an implicit selection criteria to assess which patients are deemed appropriate candidates for a laparoscopic approach. The criteria vary among institutions and surgeons in accordance with proficiency and expertise; and will evolve with time. 


\section{$\underline{\text { Section 1. Indications }}$}

\section{Topic 1: Colorectal Liver Metastases}

\section{Are Laparoscopic Liver Resections (LLR) indicated for the management of colorectal metastases (CRLM)?}

The literature suggests improved short-term outcomes for LLR of CRLM compared to Open Liver Resection (OLR) with similar long-term outcomes. A recent meta-analysis found a reduced blood loss and need for transfusion with comparable operative times and length of hospital stay in the laparoscopic group. Overall survival and disease-free survival were similar between the groups and a lower incidence of R1 resections was observed in the laparoscopic group (9). Preliminary results from the first large-scale prospective randomised control trial (COMET) ${ }^{(10)}$ comparing laparoscopic and open liver resections for CRLM has shown improved short-term outcomes for the laparoscopic approach which is supported by previous propensity score matched studies (11). Other studies report similar benefits in those aged over 70 (12). Increasing margin width in R0 resections did not significantly correlate with better overall survival (13) and as such the guidelines confirm that parenchymal sparing resections should continue to be the basis of treatment of CRLM. The guidelines conclude that with appropriate expertise, the laparoscopic approach is a valid alternative to the treatment of CRLM. See R1.1 and R1.2 (see table 1 for complete list of recommendations):

\section{What is the role of laparoscopy in the management of simultaneous colonic and liver resection for synchronous colorectal metastases?}

A laparoscopic approach was associated with a shorter hospital stay than an open approach with no difference in overall survival for patients with synchronous hepatectomy and colectomy (14). There is, however insufficient comparative data for combined major liver and colorectal resections. The experts agreed that combined 
laparoscopic major liver and colonic resections are complex and lengthy procedures with the potential for increased operative risks. However, simultaneous resections for nonrectal primaries with peripheral liver lesions requiring limited hepatectomy or left lateral sectionectomy were considered a good treatment option. Systematic review suggests that the timing of liver resection for synchronous liver metastasis should be decided according to technical and oncological considerations (15). The guidelines emphasise a need for a multidisciplinary approach to these patients. See R2.2, R2.2 and R2.3

\section{Topic 2: Benign and rare non-colorectal metastases}

What is the role of LLR in the management of benign disease and rare noncolorectal metastases?

Operative trends for benign disease demonstrate that the proportion of cases performed laparoscopically is increasing (16). LLR for benign lesions has lower intra-operative blood loss, frequency of complications, post-operative analgesic requirements, time to oral intake and a shorter hospital stay (17). With respect to neuroendocrine tumours (NETs) observational studies (18) highlight the feasibility, safety and oncological efficiency of LLR for NETs and other non-colorectal liver metastasis when clinically indicated. See R3.1 and 3.2

\section{Topic 3: Hepatocellular Carcinoma (HCC)}

\section{Is LLR indicated for the management of HCC?}

Meta-analysis and large propensity score matched studies of open versus laparoscopic liver resection for HCC have strongly suggested that LLR for HCC is associated with reduced blood loss, transfusion rate, post-operative ascites and liver failure and hospital stay with comparable operation time, disease free 
margin and recurrence rates $(19,20)$. This has been confirmed for major resections in a recent series (21). For minor resections a laparoscopic approach was found to be the only independent factor to reduce the complication rate in resections for HCC (22). See R4.1, 4.2, 4.3 and 4.4.

\section{What is the role of LLR in cirrhotic patients?}

No differences in operative time, blood loss, intraoperative complications, hospital stay and morbidity were found in LLR for cirrhotics compared to noncirrhotics (23). A laparoscopic approach appears to reduce the incidence of postoperative ascites, liver failure (24) and morbidity assessed in terms of "Comprehensive Complication Index" with no difference in overall or disease-free survival at 2 years ${ }^{25)}$. The evidence for both LLR in patients with significant portal hypertension, ascites and Child-Pugh B cirrhosis is limited to single studies $(26,27)$ and as such the guidelines recommend caution with these patient cohorts. See R5.1, 5.2 and 5.3.

\section{Topic 4: Living Donor}

What is the role of the laparoscopic technique for living donor hepatectomy (LDH)?

The evidence suggests that there is an improved quality of life with LLS for living donor hepatectomy that includes a shorter hospital stay and an earlier return to work ${ }^{28)}$. The experts discussed the differences between left lateral graft retrieval for paediatric transplantation and full right or full left hepatectomy for adult transplantation. It was highlighted that the evidence for full right and full left hepatectomy is primarily based on laparoscopic assisted procedures (hybrid) 
with only limited studies focusing on pure laparoscopic donor hepatectomy and hence minimally invasive donor major hepatectomy has not yet been standardised and should be restricted to expert centres. See 6.1, 6.2, 6.3 and 6.4. 


\section{Section 2. Patients and Complex Diseases}

\section{Topic 5: High risk patients}

\section{Are there contra-indications for LLR in elderly and high BMI patients (fragile}

patients)?

LLR for elderly patients has demonstrated lower intraoperative blood loss, hospital stay and morbidity with comparable oncological outcomes to OLR (12, 29). There are limited comparative studies regarding LLR in obese patients but evidence suggests that in selected patients it is an appropriate treatment strategy (30). See R7.1, 7.2 and 7.3

\section{Topic 6: Redo liver resections}

Are LLR feasible in patients with previous liver resection?

Evidence suggests that LLR for re-do liver surgery is an appropriate option although repeat resections have greater operative time and blood loss than primary resections $(31,32)$. The experts suggested that an initial laparoscopic resection may facilitate repeated resections by limiting the amount of adhesions, thereby providing an important advantage. See $\mathbf{R 8}$

\section{Topic 7: Technically complex settings}

\section{Is there a role for LLR in patients requiring two-stage hepatectomy?}

There are limited comparative studies specifically regarding LLR for two-stage hepatectomies. Observational studies suggests it is feasible and without detrimental effects on long-term outcomes $(33,34)$. See R9 
Is LLR feasible in patients with large lesions and lesions in close proximity to major vessels?

Reports from cohorts studies of large $(5-10 \mathrm{~cm})$ and giant $(>10 \mathrm{~cm})$ tumours suggests that the resection of such lesions can be addressed laparoscopically with no increased morbidity. However, greater operative time and blood loss was observed when compared with LLS for smaller tumours $(35,36)$. Other reports have shown that in expert hands, lesions located in close proximity to the major vasculature can be addressed laparoscopically without detrimental effects (37).

See R10.1 and 10.2. 


\section{$\underline{\text { Section 3. Procedures }}$}

\section{Topic 8: Major Hepatectomies}

What is the role of the laparoscopic technique for right hemihepatectomies?

The largest meta-analysis to date has shown that laparoscopic major hepatectomies have less blood loss, morbidity and length of stay with similar operative times, transfusion rates and completeness of resection compared to OLR (38). The expert panel suggested that the feasibility, reproducibility and implementation of left and right hepatectomies is sufficiently different that they should be considered separately. In experienced hands laparoscopic right hemihepatectomies are associated with reduced hospital stay and blood loss. Mortality and completeness of resection are comparable to an open approach $(39,40)$ See R11.1, 11.2, 11.3 and 11.4.

What is the role of the laparoscopic technique for left hemi-hepatectomies? Compared to an open approach a laparoscopic approach is associated with reduced blood loss, morbidity and hospital stay with comparable operative times, completeness of resection and mortality $(41,42)$ See to R12

Topic 9: Minor resections, resections on difficult segments, parenchymal sparing / anatomical segmentectomies

What is the role of the laparoscopic technique for minor liver resections? A meta-analysis reports lower blood loss, transfusions rates, morbidity and length of hospital stay for laparoscopic minor resections compared to open resections (38). Laparoscopic left lateral sectionectomies are consistently associated with shorter 
hospital stay when compared to the open approach (43). The evidence for a laparoscopic approach to Segments $4 \mathrm{~b}, 5$ and en bloc cholecystectomy for gallbladder cancer is limited, but suggests similar perioperative outcomes to the open approach for T1 and T2 gallbladder cancers $(44,45)$. See R13.1 and 13.2

What is the role of the laparoscopic technique for liver resections in the “difficult segments (1, 4a, 7 and 8)"?

The expert panel acknowledged that resections in these segments, especially when anatomical, are highly complex and require advanced expertise in LLS. Minor LLRs in segment 1, 4a, 7 and 8 are associated with greater operative time and blood loss than equivalent resections in the anterolateral segments. However, mortality and morbidity is not different (46). Compared to OLR, LLR is associated with reduced blood loss and hospital stay (47). A trans-thoracic approach and modified to the patient's position may be useful alternatives to the classic approach to the postero-superior segments $(48,49)$. The perioperative outcomes of robotic and laparoscopic resections of the postero-superior segments appear to be similar in terms of blood loss, hospital stay, morbidity and completeness of resection (50). See $\mathbf{R 1 4 . 1}, 14.2$ and 14.3

\section{Is LLR applicable for parenchyma-sparing procedures and anatomic}

\section{segmentectomies?}

Laparoscopic and open sectionectomies have been found to have similar perioperative outcomes ${ }^{(39)}$. Various techniques, including a Glissonian approach, staining and indocyanine green fluorescence imaging have been suggested to facilitate a true anatomical segmentectomy (51 - 53). Evidence for parenchyma- 
sparing LLR for centrally located lesions is limited. However studies document R0 and recurrence rates that fall within the average published data $(54,55)$. See R15.1 and 15.2 


\section{Section 4. Technique}

Topic 10: Minimally-invasive approaches, surgical devices, intra-operative staging and planning

What is the role of the hand-assisted technique and hybrid procedures for liver resections?

The evidence suggests that no one approach (open, hybrid, HALS or pure laparoscopic) is totally superior in terms of operative or post-operative factors but it has been suggested that HALS and hybrid techniques may serve as a bridge from open to laparoscopic surgery during the learning curve ${ }^{(56)}$. See R16

\section{What is the role of the robotic approach for liver resections?}

The robotic approach has a longer operative time and higher costs compared to a laparoscopic approach but comparable blood loss, length of stay, resection margins and morbidity $(57,58)$. Compared to an open approach, a study found total in-hospital cost to be reduced in spite of elevated operative cost (59). See R17

\section{What is the role of intraoperative ultrasound for LLR?}

The increased sensitivity of intra-operative ultrasound (compared to preoperative imaging and diagnostic laparoscopy) has been strongly suggested by numerous studies $(60,61)$. Multiple technical papers describe ultrasound as a necessary tool to investigate liver anatomy and tumour location and to plan transection lines and margins ${ }^{(62,63)}$. See R18 
What are the available techniques for parenchymal transection?

Multiple technical and comparative papers highlight the roles of differing transection devices. However there is no universal agreement regarding the optimal technique (64-66). See R19.1, 19.2 and 19.3

Topic 11: Anatomic Major resection (formal right / left hemihepatectomies)

What are the available safe techniques for inflow control during major anatomical resections?

The majority of European centres have a preference for the hilar approach, regularly demonstrating its safety and reproducibility (67). However, several centres outside of Europe report good outcomes with a Glissonian approach (68). See to R20

What are the available safe techniques during right hemi-hepatectomy? Although the anterior approach to liver transection, without prior liver mobilization, has been recommended by many a conventional approach with liver mobilization prior to transection is also possible and recommended by others. The choice between the two techniques depends on surgeon's preference, tumour size and liver fragility. Whilst the hanging manoeuvre has been used and recommended by some surgeons its reproducibility has not yet been demonstrated ${ }^{(69,70)}$. See R21.1, 21.2, 21.3, 21.4 and 21.5 


\section{Topic 12: Bleeding control / conversion}

\section{What are the haemostatic techniques during laparoscopic liver resections?}

The use of an intermittent Pringle's manoeuvre has been reported to have no detrimental effects on post-operative liver function (71). Continuous hemi-hepatic inflow control has been shown to reduce blood loss compared to an intermittent Pringle's manoeuvre with no detriment to post-operative liver function (72). Several technical papers highlight the importance of a sufficient cuff of tissue when applying clips and endovascular staplers (73). Lower intra-operative blood loss is reported in patients with a central venous pressure (CVP) lower than $5 \mathrm{cmH}_{2} \mathrm{O}$ (74). The efficacy of stroke volume variation as an alternative to CVP monitoring has been demonstrated (75). See R22.1, 22.2, 22.3 and 22.4

\section{When and how should conversions to open surgery be considered?}

Conversion during LLR is associated with higher post-operative morbidity however in comparison to planned OLR the outcomes were found to be similar (76). Risk factors for conversion include an increasing BMI, tumour size and resection extent as well as resections in the postero-superior segments and cirrhosis (36 77, 78). In the case of conversion for significant vascular injury, temporary control of the bleeding source prior to conversion is highly recommended. See R23.1, 23.2 and 23.3 


\section{Section 5. Implementation}

\section{Topic 13: Surgeon / centre / learning curves}

What training and preparation should surgeons pursue before performing minor, major and complex liver resections?

With experience both operative time and blood loss decreases $(79,80)$ and experience gained during minor resections may shorten the learning curve for major resections (81). The learning curve for minor resections is suggested to be 60 cases (79) and that for major resections is 55 (having already developed experience on minor resections) ${ }^{(82) . ~ S e e ~ R 24.1, ~ 24.2, ~} 24.3$ and 24.4

\section{Which centres should be performing laparoscopic liver resections?} Laparoscopic liver surgery should not be developed in isolation from an open liver program. Major and complex LLS should be gradually implemented with increasing collective expertise for safe patient selection and management. (83) See R25.1, 25.2 and 25.3

Should laparoscopic liver resection become adopted in all liver surgical centres?

A meta-analysis has found that the laparoscopic approach offers fewer complications, decreased blood loss and a shorter hospital stay with comparable oncological outcomes in selected patients (38). Therefore the guidelines confirm that all centres should implement a program of LLS and offer it to patients with the appropriate indications according to the local level of proficiency. Ideally, at least two surgeons proficient in LLS in each centre is recommended. See R26 


\section{Topic 14: Training / Registries}

\section{Who should be undertaking training and mentoring roles in LLR?}

With regards to trainers / mentors and registries / learned societies no evidence based studies are available. However, the learning curve for minor resections can significantly reduced by surgeons assisting one another ${ }^{84)}$. The recommendation of the experts is that mentors and trainers must be experienced surgeons with a current and up-to-date knowledge of the literature, whilst registries are necessary for evaluation of LLR and individual surgeons / centres alike. This relates to R27, R28 and R29 


\section{Discussion:}

The European Guidelines Meeting for Laparoscopic Liver Surgery was devised to produce specific guideline statements to ensure the safe progression and dissemination of laparoscopic liver surgery. The guidelines produced further the work of the previous consensus meetings by providing specific guidance to both expert and training laparoscopic liver surgeons. The 67 guidelines combine the most up-to-date evidence with expert opinion to guide the dissemination of laparoscopic liver surgery. Each guideline reached at least a 95\% consensus amongst the expert committee before its acceptance into the meeting. During the meeting each statement was opened to a vote by all those in attendance (228 surgeons including the faculty). The median agreement was 88\% (with at least 160 surgeons responding to each vote) demonstrating the support of these guidelines by those with a special interest in laparoscopic liver surgery. All statements were approved and endorsed by the independent validation committee.

The EGMLLS explored new areas in the application of laparoscopy in an everincreasing cohort of patients and provided guidance to the appropriateness of LLR for specific diseases. Indications have been refined taking into account specific subcategories of high-risk patients and technically complex disease. Moreover, the guidelines re-define the classification of resections adding "technically major" resections, such as those in the postero-superior segments, to the established anatomical minor and major resections. Specific scenarios that require more experience were highlighted with the guidelines advocating caution dependent on the surgeon's expertise and available technical equipment. 
The Southampton Guidelines state that when performed by expert surgeons, LLR offers significant advantages in terms of a reduced risk of post-operative ascites and liver decompensation in patients with cirrhosis. For patients with CRLM LLR was deemed an appropriate option that offers significant benefits in terms of a shorter hospital stay and lower complication rate. However, the need to adhere to a parenchymal sparing approach was stressed. The use of LLR for living donor hepatectomy is limited to a few highly specialised centres worldwide but may now be regarded as standard practice for left lateral sectionectomy in adult-topaediatric donation.

The Southampton Guidelines advocate that the laparoscopic approach should be considered standard practice for lesions in the left lateral and the anterior segments. The guidelines state that in expert hands, LLR for lesions in the posterosuperior segments may maintain the advantages seen in the anterolateral segments. Subcategories of "high-risk" patients, such as the elderly and patients with high BMI, were no longer considered as contra-indications to LLR. Technically challenging resections such as repeat resections or 2 stage hepatectomies, resections for large lesions and lesions in close proximity to the hilum are now considered possible by surgeons with extensive experiences in LLS.

The Southampton Guidelines highlight the difference in difficulty and outcomes between laparoscopic left and right hemi-hepatectomies. Hence, it was advised that their uptake occur at different points in the learning curve. Regarding inflow control and parenchymal transection the guidelines state that the choice of 
technique is dependent on the characteristics of the disease and the surgeon's preference. Pringle's Manoeuvre and the management of intra-vascular volume to provide a low CVP are both essential to reduce blood loss during transection. And, as in open liver surgery the need for intra-operative ultrasound was considered essential.

The guidelines regarding the implementation of LLS are of paramount importance in the EGMLLS. A background in open liver surgery and advanced laparoscopic skills prior to starting LLR are considered essential. The guidelines recommend fellowships, courses and proctored programmes to facilitate the training and development of laparoscopic liver surgeons. These fellowships should be conducted in established, high-volume centres that routinely perform minor, major and complex major resections. Those providing supervision, as mentors and proctors, should themselves have already reached competency and are thus able to provide safe guidance during the training of less experienced surgeons. Importantly, it was recommended that each specialist centre should offer a laparoscopic approach as part of its multi-disciplinary management of liver disease and should ideally have a minimum of two surgeons competent in LLS to support, assist and critique each other to aid development.

It is important to note that the majority of the evidence used in the production of these guidelines report data from specialist liver centres, which may represent a publication bias. However, this factor is of critical importance, as these guidelines should not be misconstrued as an invitation to begin performing laparoscopic liver surgery in the absence of experience and support. The authors must once 
again stress that laparoscopic liver surgery is complex and requires advanced laparoscopic skills, comprehensive experience of open liver surgery and the support of an experienced team. Finally, the terms "experienced surgeons" and "selected patients" are not simple, rigid definitions but represent a malleable spectrum where multiple confounding factors, which will evolve with time and vary between centres, must be considered. Although previous manuscripts have suggested that between 20 to 60 minor resections and 30 to 60 major resections (having already reached competency with minor resections) are required to overcome the learning curve (79-82) the expert panel were in agreement that no specific number can be given to the number of resections performed for a surgeon to reach "competency" and patient factors must be weighed with respect to the experience of the surgeon and their team.

With the exponential growth of laparoscopic liver surgery it will no doubt be necessary to review the current guidance with the passage of time to ensure that they continue to represent the most contemporary and highest level of evidence available in order to provide safe guidance in the dissemination of laparoscopic liver surgery. 


\section{Conclusion:}

The European Guidelines Meeting for Laparoscopic Liver Surgery has produced a set of clinical practice guidelines that have been independently validated for the safe development and progression of laparoscopic liver surgery. Using a robust methodology the Southampton Guidelines have amalgamated the available evidence and a wealth of experts' knowledge taking in consideration the relevant stakeholders' opinions and complying with the international methodology standards. These guidelines are not an endorsement for a novice to perform LLS without the appropriate training and ideally LLS should be performed within the confines of an institution with an established support network and experience in liver surgery. 


\section{References:}

1. Buell, Cherqui, Geller et al. The International Position on Laparoscopic Liver Surgery: The Louisville Statement, 2008. Ann Surg. 2009; 250: 825-830.

2. Wakabayashi, Cherqui, Geller et al. Recommendations for Laparoscopic Liver Resection: A report from the second international consensus conference held in Morioka. Ann Surgery; 261: 619-629.

3. Cherqui. Evolution of laparoscopic liver resection. BJS. 2016; 103(11): 1405-1407.

4. Abu Hilal. Why do we need guidelines in laparoscopic liver surgery? HPB. 2017; epublication ahead of press.

5. sign.ac.uk

6. Dalkey. Delphi. RAND coporation. 1967

7. agreetrust.org

8. Brouwers, Kho, Browman et al. The Global Rating Scale complements the AGREE II in advancing the quality of practice guidelines. J Clin Epidemiol. 2012;65:526-534

9. Luo, Yu, Bai. Laparoscopic Hepatectomy for Liver Metastases from Colorectal Cancer: A Meta-analysis. Journal of Laparoendoscopic \& Advanced Surgical Techniques. 2014 Apr;24(4):213-22.

10. Clinicaltrials.gov 
11. Cipriani, Rawashdeh, Stanton et al. Propensity score-based analysis of outcomes of laparoscopic versus open liver resection for colorectal metastases. BJS. 2016; 103: 1504-1512.

12. Martínez-Cecilia, Cipriani, Vishal et al. Laparoscopic Versus Open Liver Resection For Colorectal Metastases in Elderly and Octogenarian Patients. Annals of Surgery. 2017 Feb;:1-9.

13. Montalti, Tomassini, Laurent et al. Impact of surgical margins on overall and recurrence-free survival in parenchymal-sparing laparoscopic liver resections of colorectal metastases. Surgical Endoscopy. Springer US; 2016 Jul 5;:1-12.

14. Wei, He, Wang et al. Laparoscopic versus Open Hepatectomy with or without Synchronous Colectomy for Colorectal Liver Metastasis: A Meta-Analysis. Katoh M, editor. PLoS ONE. 2014 Jan 29;9(1):e87461-9.

15. Lupinacci, Andraus, De Paiva Haddad et al. Simultaneous laparoscopic resection of primary colorectal cancer and associated liver metastases: a systematic review. Tech Coloproctol. 2013 Sep 21;18(2):129-35.

16. Kim, Amini, He et al. National trends in the use of surgery for benign hepatic tumours in the United States. Surgery. 2015: 157(6). 1055-1064.

17. Croome and Yamashita. Laparoscopic vs open hepatic resection for benign and malignant tumours: an updated meta-analysis. Arch Surg. 2010: 145(11), 1109-1118.

18. Kandil, Noureldine, Koffron et al. Outcomes of laparoscopic and open resection for neuroendocrine liver metastatases. Surgery. 2012: $152(6), 1225-1231$. 
19. Xiong, Altaf, Javed et al. Meta-analysis of laparoscopic vs open liver resection for hepatocellular carcinoma. World Journal of Gastroenterology. 2012; 18(45): 6657-6668.

20. Takahara, Wakabayashi, Beppu et al. Long-term and perioperative outcomes of laparoscopic versus open liver resection for hepatocellular carcinoma with propensity score matching: a multiinstitutional Japanese study. J Hepatobiliary Pancreat Sci. 2015; 22: 721727.

21. Yoon, Kim, Kang et al. Pure Laparoscopic Versus Open Right Hepatectomy for Hepatocellular Carcinoma in Patients With Cirrhosis: A Propensity Score Matched Analysis. Ann Surg 2016. Epub ahead of press.

22. Sposito, Battiston, Facciorusso et al. Propensity score analysis of outcomes following laparoscopic or open liver resection for hepatocellular carcinoma. Br J Surg. 2016 Jun;103(7):871-80.

23. Shehta, Han, Yoon et al. Laparoscopic liver resection for hepatocellular carcinoma in cirrhotic patients: 10-year single-center experience. Surg Endosc. 2016.

24. Zhang, Huang, Chen et al. A Comparison of Laparoscopic Versus Open Left Hemihepatectomy for Hepatocellular Carcinoma. Surg Laparosc Endosc Percutan Tech. 2016 Apr;26(2):146-9.

25. Morise, Ciria, Cherqui, et al. Can we expand the indications for laparoscopic liver resection? A systematic review and meta-analysis of laparoscopic liver resection for patients with hepatocellular carcinoma and chronic liver disease. J Hepatobiliary Pancreat Sci. 2015. 
26. Harada, Maeda, Yoshizumi et al Laparoscopic Liver resection is a feasible treatment for patients with hepatocellular carcinoma and portal hypertension. Anticancer Res. 2016; 36(7): 3489-3497.

27. Cai, Liang, Tunan et al. Liver cirrhosis grading Child-Pugh B: a Goliath to challenge in laparoscopic liver resection? Prior experience and matched comparisons. Hepatobiliary Surg Nutr. 2015; 4(6): 391-397.

28. Samstein, Griesemer, Cherqui et al. Fully laparoscopic left-sided donor hepatectomy is safe and associated with shorter hospital stay and earlier return to work: A comparative study. Liver Transplantation. 2015; 6: 768-773.

29. Cauchy, Fuks, Nomi et al. Benefits of laparoscopy in elderly patients requiring major liver resection. JACS 2015;222:174-84

30. Uchida, Iwashita, Saga et al. Benefit of laparoscopic liver resection in high body mass index patients. World J Surg 2016;22:3015-22

31. Cioffi, Belli, Fantini et al. Repeat liver surgery by laparoscopy for a malignant recurrence after previous open or laparoscopic resection. Hepatoma Res 2015;1

32. Shelat, Serin, Samim et al. Outcomes of repeat laparoscopic liver resection compared to the primary resection. World J Surg $2014 ; 38: 3175-80$

33. Fuks, Nomi, Ogiso et al. Laparoscopic two-stage hepatectomy for bilobar colorectal liver metastases. BJS 2015;102:1684-90

34. Di Fabio, Whistance, Rahman et al. Exploring the role of laparoscopic surgery in two-stage hepatectomy for bilobar colorectal lover metastases. J Laparoendosc Adv Tech A. 2012; 22(7): 647-650. 
35. $\mathrm{Ai}, \mathrm{Li}$, Chen et al. Feasibility and safety of laparoscopic liver resection for hepatocellular carcinoma with a tumor size of 5-10 cm. PLOS One 2013;8:723-28

36. Shelat, Cipriani, Basseres et al. Pure laparoscopic liver resection for large malignant tumors: does size matter? Ann Surg Oncol 2014;22:1288-93

37. Yoon, Han, Cho et al. Laparoscopic liver resection for centrally located tumors close to the hilum, major hepatic veins, or inferior vena cava. Surg 2013;153:502-9

38. Ciria, Cherqui, Geller et al. Comparative Short-term Benefits of Laparoscopic Liver Resection: 9000 Cases and Climbing. Annals of Surgery. 2016: 263(4), 761-777.

39. Takahara, Wakabayashi, Konno et al. Comparison of laparoscopic major hepatectomy with propensity score matched open cases from the National Clinical Database in Japan. J Hepatobiliary Pancreat Sci. $2016 ; 23: 721-734$

40. Abu Hilal, Di Fabio, Teng et al. Single-centre comparative study of laparoscopic versus open right hepatectomy. J Gastrointest Surg. 2011;15:818-823.

41. Ye, Ni, Zhou et al. Laparoscopic versus open left hemihepatectomy for hepatolithiasis. J Surg Res. 2015;199:402-406.

42. Namgoong, Kim, Park et al. Comparison of laparoscopic versus open left hemihepatectomy for left-sided hepatolithiasis. Int J Med Sci. 2014;11:127-133. 
43. Ding, Cai and Qin. Pure Laparoscopic Versus Open Liver Resection in Treatment of Hepatolithiasis Within the Left Lobes: A Randomized Trial Study. Surg Laparosc Endosc Percutan Tech. 2015;25:392-394.

44. Agarwal, Javed, Kalayarasan et al. Minimally invasive versus the conventional open surgical approach of a radical cholecystectomy for gallbladder cancer: a retrospective comparative study. HPB (Oxford). 2015;17:536-541.

45. Itano, Oshima, Minagawa et al. Novel strategy for laparoscopic treatment of pT2 gallbladder carcinoma. Surg Endosc. 2015;29:36003607.

46. Lee, Han, Yoon et al. Comparison of laparoscopic liver resection for hepatocellular carcinoma located in the posterosuperior segments or anterolateral segments: A case-matched analysis. Surgery. $2016 ; 160: 1219-1226$

47. Scuderi, Barkhatov, Montalti et al. Outcome after laparoscopic and open resections of posterosuperior segments of the liver. Br J Surg. 2017 Feb 13. doi: 10.1002/bjs.10489. [Epub ahead of print]

48. Chiow, Lewin, Manoharan et al. Intercostal and transthoracic trocars enable easier laparoscopic resection of dome liver lesions. HPB (Oxford). 2015;17:299-303.

49. Ogiso, Conrad, Araki et al. Laparoscopic Transabdominal With Transdiaphragmatic Access Improves Resection of Difficult Posterosuperior Liver Lesions. Ann Surg. 2015;262:358-365. 
50. Montalti, Scuderi, Patriti et al. Robotic versus laparoscopic resections of posterosuperior segments of the liver: a propensity score-matched comparison. Surg Endosc. 2016;30:1004-1013

51. Machado, Surjan, Basseres et al. The laparoscopic Glissonian approach is safe and efficient when compared with standard laparoscopic liver resection: Results of an observational study over 7 years. Surgery. 2016;160:643-651.

52. Sakoda, Ueno, Iino et al. Anatomical laparoscopic hepatectomy for hepatocellular carcinoma using indocyanine green fluorescence imaging. J Laparoendosc Adv Surg Tech A. 2014;24:878-882

53. Ishizawa, Zuker, Kokudo et al. Positive and negative staining of hepatic segments by use of fluorescent imaging techniques during laparoscopic hepatectomy. Arch Surg. 2012;147:393-4

54. Cipriani, Shelat, Rawashdeh et al. Laparoscopic Parenchymal-Sparing Resections for Nonperipheral Liver Lesions, the Diamond Technique: Technical Aspects, Clinical Outcomes, and Oncologic Efficiency. J Am Coll Surg. 2015;221:265-272

55. Conrad, Ogiso, Inoue et al. Laparoscopic parenchymal-sparing liver resection of lesions in the central segments: feasible, safe, and effective. Surg Endosc. 2015;29:2410-2417

56. Hasegawa, Koffron, Buell et al. Approaches to laparoscopic liver resection: a meta-analysis of the role of hand-assisted laparoscopic surgery and the hybrid technique. J Hepatobiliary Pancreat Sci 2015; 22(5):335-341. 
57. Qiu, Chen and Chengyou. A systematic review of robotic-assisted liver resection and meta-analysis of robotic versus laparoscopic hepatectomy for hepatic neoplasms. Surg Endosc 2016; 30(3):862-875.

58. Troisi, Patriti, Montalti et al. Robot assistance in liver surgery: a real advantage over a fully laparoscopic approach? Results of a comparative bi-institutional analysis. Int J Med Robot 2013; 9(2):160166.

59. Sham, Richards, Seo et al. Efficacy and cost of robotic hepatectomy: is the robot cost-prohibitive? J Robot Surg 2016.

60. Vigano, Ferrero, Amisano et al. Comparison of laparoscopic and open intraoperative ultrasonography for staging liver tumours. Br J Surg. $2013 ; 100(4): 535-542$.

61. Milsom, Jerby, Kessler et al. Prospective, blinded comparison of laparoscopic ultrasonography vs. contrast-enhanced computerized tomography for liver assessment in patients undergoing colorectal carcinoma surgery. Dis Colon Rectum 2000; 43(1):44-49.

62. Aldrighetti, Belli, Boni et al. Italian experience in minimally invasive liver surgery: a national survey. Updates Surg 2015; 67(2):129-140.

63. Cherqui, Figueroa and Gelli. Tips of totally laparoscopic left hepatectomy.J Hepatobiliary Pancreat Sci 2016; 23(2):E1-4.

64. Berber, Akyuz Aucejo et al. Initial experience with a new articulating energy device for laparoscopic liver resection. Surg Endosc 2014; 28(3):974-978.

65. Buell, Gayet, Han et al. Evaluation of stapler hepatectomy during a laparoscopic liver resection. $H P B$ (Oxford) 2013; 15(11):845-850. 
66. Dural, Akyuz, Yazici et al. Safety and Efficacy of a New Bipolar Energy Device for Parenchymal Dissection in Laparoscopic Liver Resection. Surg Laparosc Endosc Percutan Tech 2016; 26(1):21-24.

67. Tzanis, Shivathirthan, Laurent et al. European experience of laparoscopic major hepatectomy. J Hepatobiliary Pancreat Sci 2013; 20(2):120-124.

68. Machado, Surjan, Basseres et al. The laparoscopic Glissonian approach is safe and efficient when compared with standard laparoscopic liver resection: Results of an observational study over 7 years. Surgery 2016.

69. Soubrane, Schwarz, Cauchy et al. A Conceptual Technique for Laparoscopic Right Hepatectomy Based on Facts and Oncologic Principles: The Caudal Approach. Ann Surg 2015; 261(6):1226-1231.

70. Dokmak, Ben Safta, Fteriche et al. Pure laparoscopic right hepatectomy with the hanging maneuver for multiple hepatocellular adenomas. Ann Surg Oncol 2014; 21(12):3800-3801.

71. Dua, Worhunsky, Hwa et al. Extra-corporeal Pringle for laparoscopic liver resection. Surg Endosc. 2015: 29(6), 1348-1355.

72. Zhang, Yang, Deng et al. Intermittent Pringle's manoeuvre versus continuous hemihepatic vascular inflow occlusion using extraglissonian approach in laparoscopic liver resection. Surg Endosc. 2016: 30(3), 961-70.

73. Abu Hilal, Underwood, Taylor et al. Bleeding and haemostatis in laparoscopic liver surgery. Surg Endosc. 2009. 24: 572-277.

74. Jones, Moulton and Hardy. Central venous pressure and its effect on blood loss during liver resection. Br J Surg. 1998: 85(8), 1058-1060. 
75. Ratti, Cipriani, Reineke et al. Intraoperative monitoring of stroke volume variation versus central venous pressure in laparoscopic liver surgery: a randomized prospective comparative trial. HPB. 2016: 18(2), 136-144.

76. Cauchy, Fuks, Nomi et al. Risk Factors and consequences of conversion in laparoscopic major liver resection. Br J Surg. 2015: 102(7), 785-795.

77. Ratti, D'alessandro, Cipriani et al. Influence of Body Habitus on Feasibility and Outcome of Laparoscopic Liver Resections: A Prosective Study. J Hepatobiliary Pancreat Sci. 2016: 23, 373-381.

78. Troisi, Montalti, Van Limmen et al. Risk factors and management of conversions to an open approach in laparoscopic liver resection: analysis of 265 consecutive cases. HPB. 2014: 16, 75-82.

79. Vigano, Laurent, Tayar et al. The learning curve in Laparoscopic Liver Resection: Improved Feasibility and Reproducibility. Annals of Surgery. 2009: 250 (5), 772-782.

80. Dagher, Gayet, Tzanis et al. International experience for laparoscopic major liver resection. J Hepatobiliary Pancreat Sci. 2014: 21(10), 732736.

81. Hasegawa, Nitta, Takahara et al Safely extending the indications of laparoscopic liver resection: When should we start laparoscopic major hepatectomy? Surg Endosc. 2016.

82. Van der Poel, Besselink, Cipriani et al. Outcome and learning curve in 159 consecutive patients undergoing total laparoscopic hemihepatectomy. JAMA Surg. 2016. 
83. Kluger, Vigano, Barroso et al. The learning curve in laparoscopic major liver resection. J Hepatobiliary Pancreat Sci. 2013: 20(2), 131-136.

84. Goh, Chan, Wong et al. Factors associated with and the outcomes of open conversion after laparoscopic minor hepatectomy: Initial experience at a single instituation. Surg Endosc. 2015: 29(9), 2636-2642. 